\title{
Musical Meaning ANd the Musicology of Record PROduction ${ }^{1}$
}

\author{
Simon Zagorski-Thomas
}

\section{Introduction}

The study of record production, and indeed of musicology in general, tends to divide into two camps that we might broadly describe as production and reception based approaches. On one side academics study the technical and creative process that shapes the musical output and on the other they study the way in which audiences (real or idealised) engage with the »text « to create meaning. This paper will attempt to bridge some of the gaps between these two areas of study. One of the crucial aspects of this is the distinction between a single universal and inherent meaning in a piece of music and an infinite range of potential individual interpretations that is often associated with »post-structuralist « approaches ${ }^{2}$. Many of these ideas are applicable more widely - both to musical analysis and creativity in general - but in this article I will be looking specifically at the production and reception of recorded music.

1 This article is part of a bigger research project that has included several conference papers and articles (Zagorski-Thomas 2008a, 2008b, 2009, 2010), panel discussions at the 2005 and 2010 Art of Record Production conferences, and the preparation of a monograph on the Musicology of Record Production which is currently underway. The music and text of my PhD portfolio submission can be accessed at http://www.zagorski-thomas.com. This particular aspect of the project stems from Professor Allan Moore's comments at my PhD viva concerning the attribution of meaning through musical analysis. When this article was originally presented at the ASPM symposium Black Box Pop: Analysen Populärer Musik in Mannheim in November 2010, it included seven recorded examples, listed in the discography below. Excerpts from all of these can be listened to on iTunes and all but the Darkthrone track are available on Spotify.

2 Although this is mostly a position ascribed by critics to authors who would both reject the position and the »post-structuralist « label. 


\section{The Theoretical Context}

Allan Moore's comments and questions at my $\mathrm{PhD}$ viva concerned the problem of reconciling the composer/producer's intentions and the listener/ audience's responses. There is no single way to interpret a piece of music and this gives rise to the question of whether, and how, one analysis can be given greater credence or validity than another. As part of this wider discussion I want to examine the idea that there may be some universality of meaning in sound for us as humans. I will also make suggestions how we may analyse and discuss any broadly held, culturally constructed, agreements about meaning in musical sound that may exist in various social groupings.

The first musical example is "You Can't Always Get What You Want « by the Rolling Stones (1969). The key feature of this track that informs this discussion is the sound of the choir and, in particular, the sound of the space they are in (or appear to be in) and the voice types. I would expect the vast majority of potential readers of this article to associate this sound with a Christian choir in a church. There are two aspects of this interpretation, however: firstly there are what I will characterise as the ecological, universal or centripetal forces upon interpretation and secondly there are the cultural, specific or centrifugal ones. Some aspects of the world as they are perceived through our bodies are immutable and universal because the experience of existing within a human body forces some types of interpretation upon us. The perception of what types of voices, what kind of energy is being expended and what kind of environment they are singing in can be seen in this light. In addition to this, for example in the interpretation of this sound as a choir in a church (or Olympic Studios) some of our perceptions rely on more culturally specific knowledge and experience. Before we explore these ideas any further, I will provide a brief survey of the academic work that has informed this analysis.

Eric Clarke (2005) has taken James Gibson's (1979) ecological approach to perception and applied it to musical analysis. This approach takes as its starting point the notion that our perceptual systems are tuned for specific forms of interpretation relating to how our bodies exist in the physical environment: for example to notice location, movement or difference. At a higher level, this transforms into the perception of the type of environment, the type of agent, the type of activity; and higher still, into meaning such as possible threat or safety. This leads to the notion that particular forms of stimulus and environment produce »inevitable tation. Thus, for example, a combination of delay and volume differences 
between signals reaching my ears will engender a »universal« interpretation of a sound source's direction: one of the basic tests of »normality « in an infant is to see if they turn towards a sound source correctly. Similarly, the amount of high frequency content and the balance of direct and ambient sound can be related to the perception of proximity and the low frequency content and reverberation time of ambience are associated with the perceived size of an enclosed space. The »universal« nature of these interpretations, of course, relies on particular features of a human form which may not always be present: two fully functioning ears for example. It also says nothing about the extent to which these attributes of human perception are inherent or learned.

George Lakoff and Mark Johnson (1980) assert that certain metaphorical relationships are inherent in our embodied existence and experience as humans within a perceptual system. These inherent relationships or interpretations are based on the kind of »universal« human embodied experience that can be found in Gibson and Clarke's ecological approach outlined above. Lakoff and Johnson use the term »schema« to describe the interpretive structures that form the basis of these metaphorical relationships. For example, the balance schema describes a physical experience, the haptic sensation of balance, that we utilise in a metaphorical sense: the balance of power, the balance of trade, and more relevantly to this study, the sound engineer's notion of balancing sounds in a recording. Lakoff and Johnson characterise the whole of human mental activity as being based on metaphor: both the primary metaphoric relationships between perceived events and actions and our embodied experience and the secondary metaphoric relationships between perceived events and actions and other metaphoric interpretations. For example, the sentence, »He was shunted from one department to another « relies on a metaphor with goods transportation by railways and our understanding of that comes, in turn, from a complex web of metaphorical relationships with our own embodied experiences of movement and agency.

A third theoretical thread in this analysis stems from Michael Bakhtin's (1981) notion of heteroglossia in literary theory. This refers to the way in which a language can be seen to be constructed from many voices, such as:

»social dialects, characteristic group behavior [sic], professional jargons, generic languages, languages of generations and age groups, tendentious languages, languages of the authorities, of various circles and of passing fashions« (Bakhtin 1981: 262). 
Authors such as Ingrid Monson (1996: 99) have applied this idea to musical analysis. One key element in this approach is the notion of centrifugal and centripetal forces acting on individuals and communities (of various sizes) in both language and creative practice. Certain cultural meanings become embedded in the experience of communities - Christians and the church choir for example - and these become part of the rules and the recognized valid examples of practice and interpretation. Centripetal forces are about conformity and unity within a community whilst centrifugal forces are about difference and individuality. A form of language or creative practice can be centrifugal in one community and centripetal in another and, conversely, an individual may engage in activities (or forms of language) of which some are centripetal and some are centrifugal to a community.

In order to give further substance to these notions of centripetal and centrifugal forces in musical language, I'm drawing on some work from systems theory to help define the idea of a »community«. Mihaly Csikszentmihalyi (1999) defined social systems of creativity as a domain of symbolic rules influencing the individual whose creative output is recognized and accepted by a field of experts (and an audience). Phillip Maclntyre (2007) has applied these ideas to the topic of record production. Within this system, the individual can be seen to react in either a centrifugal or centripetal manner (or indeed a complex mix of the two) to the rules, conventions and traditions of the cultural domain and to the critical judgment of the various members of the social field. As we will see, if we incorporate the audience into the field of the creative system this allows us to bridge the gap between production and reception approaches.

In the following sections I will utilise some further musical examples to illustrate some of the theoretical constructs used in this analytical method and which will hopefully provide a range of tools which can help to negotiate the dichotomy between a universal inherent meaning and an infinite range of potential individual interpretations.

\section{Subject Position}

Clarke (2005) has applied the term »subject position«, taken from film studies, to musical analysis: the idea that certain production decisions skew the listeners towards a particular interpretation. On a very basic level, the implied spatial positioning that comes from mixing separately recorded elements in a particular way also implies a hierarchy of musical importance. In the example of »Thoughts Of Mary Jane« (Nick Drake 1970) we have an 
example where multiple performances, recorded on multi-track tape at different times, have subsequently been mixed together to produce a stereo recording. The staging of these multiple performances places them in an imaginary spatial relationship with each other based on their placement in the stereo field and their relative volumes. ${ }^{3}$ As a general rule, the production of singer-songwriters foregrounds acoustic guitar and the voice to assert their individual agency and importance. To return to the film studies analogy, it's the aural equivalent of placing the singer-songwriter front and centre in the shot frame with the other musicians behind or to the side: the staging »forces « an interpretation upon us about which are the most important elements in this recording. Having said that, the »strength « (in terms of both volume and perceived proximity ${ }^{4}$ ) of the strings on Nick Drake albums has led fans to express dismay that Joe Boyd »over-produced« Nick Drake ${ }^{5}$.

\section{Systems Model: Cultural Domains and Social Fields}

The production »language « of the next example draws on several communities, or the cultural domains and social fields of popular music. »Party At A Rich Dude's House « (Ke\$ha 2010) draws on well established conventions from a variety of creative »systems « that exist within the stylistic groupings of popular music. The vocal and kick drum are heavily processed and distinctly foregrounded in a manner that is stereotypical of dance oriented pop music. These conventions can be viewed as central to the »rules « of the cultural domain of mixing dance oriented pop music and as flowing from two distinct characteristics of audience engagement in the social field: the first is its use as dance music and the second is its use as background listening. Mixing practice for dance music has, since the 1970s, seen a trend of foregrounding the drum kit, and particularly the kick drum, and staging it with little or no ambience (Zagorski-Thomas 2010: 254f.). This practice has

3 For the sake of clarity I'm going to ignore the further complications that recorded or added ambience and equalisation can add. This example was deliberately chosen because those variables don't contribute much to the staging of this track.

4 The perceived proximity is affected not only by the volume but also by the relative volumes of the direct and ambient sound and by the relative intensity of the high frequency content of the sound.

5 See for example http://www.nickdrake.com/talk/viewtopic.php?t=488\&sid= 6a5bf0b797b748346e7e2abbd3f677aa (accessed February 28, 2011). 
developed because its function as dance music relies on the clear perception of the attack transients, and ambience not only blurs this perception but the playback of dance music through powerful sound systems in large clubs adds a secondary playback ambience in the venue which would add further ambience to any recorded ambience and further blur this perception. The inclusion of the distorted guitar sound of punk rock in this track marks a reference to another stylistic »system «. This characteristic sound is made less significant as a genre marker by being mixed further back in the track than it would if it really aspired to be embraced by that particular social field. There's also an interesting third reference in this track when Ke\$ha sings along with the guitar line in the way that teenage audiences often do: »Dananana na nana« after the first chorus at around 1'02". Ke\$ha steps out of the role of performer and into the role of fan by following the domain rules of the audience.

\section{Centrifugal and Centripetal Social Forces}

The next example is »Transilvanian Hunger « by Darkthrone (1994) which utilises a deliberately »lo-fi « approach to recording which sounds like, and very probably is, a recording with one or two microphones of the band playing in a rehearsal room or similarly acoustically untreated space. Certain forms of mediation can be seen as representative of, or associated with, certain communities and thus acquire an ideological meaning about belonging to or rebelling against these communities. In this example, the mediation of electric guitar tones through harmonic distortion is a marker for a stylistic belonging to rock in general and to a particular metal genre. The use of quite highly sophisticated guitar amplification technology displays a centripetal attitude to the mediated sonic language of heavy metal rock music and a willingness to be seen as »belonging « to that sort of community. On the other hand, the sounds of what we might call »high fidelity « or professional recording studio practice are rejected because of an ideological association with commercialism. In this instance, Darkthrone are using the lo-fi sound as a marker of their centrifugal attitude towards the norms of professional recording practice: their unwillingness to belong to (or at least conform to the norms of) the world of commercial recorded music. 


\section{»Universal« Forms of Meaning}

This next example aims to highlight ecological and metaphorical forms of meaning that are more or less universal. This involves bodily based metaphor and perceptual forms that stem from the experience of being a human being in the world. This would include spatial perception about distance and environment types; social interpretations of perceptual experience grounded in basic human reactions like fear, excitement, passivity, relaxation etc. Before the development of electrical sound recording these types of staging were generally not controllable other than by choosing a venue type and configuring the musicians within it in a particular way: recorded music has changed that. In Lisa Hannigan's »I Don't Know« (2009) the staging has been constructed to encourage the impression of an easy going, relaxed performance and an intimate atmosphere. The gestures and activities that make these musical sounds have a recognisably low level of energy expenditure and the processing and manipulation that has been applied to them reinforces that effect. Low impact sounds are highlighted as such through the control of their frequency content: the frequencies that are associated with the sound of low intensity performance are highlighted and those of high energy performance are attenuated. For the most part this means increasing high frequency content and decreasing low frequencies for example a snare drum sound would increase in low frequency content as well as volume as it was hit harder. In this example equalisation and/or filters have been used to remove any low frequencies. With the bass sound, on the other hand, the high frequency slap of a string hitting a fret board is filtered out reducing the sonic footprint of energy expenditure and enhancing the impression of a light and easy performance. All of this highlights the atmosphere of a low energy, almost effortless performance through the medium of these basic perceptual and metaphorical connections between frequency content and performance intensity: metaphorical connections that stem from our basic experience of what sorts of noises are produced by light tapping as opposed to heavy thumping. At the same time, the presence of heightened high frequency content such as the breathiness of the voice and the »fingeriness « of the pizzicato violin create a sensation of intimacy because, once again, the everyday experience of the high frequency content of sound dissipating with distance can be used to make something seem closer than the loudspeaker it emanates from. And that sense of physical proximity translates into a perception of social proximity or intimacy through the metaphorical workings of our mind. 


\section{Cultural References}

These types of metaphorical meaning extend beyond the ecological or »universal « meanings discussed in the previous section into cultural references like the sound of familiar concert hall and performance venue types. They also extend to the sound of familiar forms of technological mediation: early recordings, vinyl crackle, telephones etc. Portishead's »Biscuit« (1994) demonstrates how this band deliberately recontextualise their live performances in the studio to reference the sound of DJ, dance music and hip hop culture. Their own musical performance is sampled, pressed onto vinyl and then re-performed through sequencing and scratching techniques. The noncontinuous sound of the vinyl crackle on the sample and the scratching of the vocal performance in this example both provide cultural references to the sound of a DJ playing back and manipulating a vinyl record. This not only marks out Portishead as belonging to a particular musical world or culture, but also demonstrates their standing through their virtuosity in these DJ related creative practices.

\section{Communal Agency And Audience Reception}

Georgina Born (2005: 20-23) draws on the work of anthropologist Arthur Gell to discuss distributed creativity and she identifies four kinds of dispersal of creative agency:

1. Retention and protension of works across time: to include influences from the past and into the future.

2. Across space: in the sense that the objectivisation of sound through recording allows multi-spaced creativity.

3. Between persons: perhaps the most obvious in that collaborative activity involves multiple agents.

4. Between subjects and objects: in the sense that the design of instruments and other tools constitutes a collaboration with their users.

How then can this be related to an attempt to reconcile production and reception based analysis? One approach is to investigate how and when the audience becomes part of the collaborative community. For example, it often seems that when artists describe a new album as "going back to their roots « they often seem to mean that they are responding to audience de- 
mand - repeating a formula that was successful - but there could be many more subtle interplays at work as well. Are they responding to what the producer or the record company perceive or imagine to be the audience demand? Are they responding to what their audience demanded when they started out or what they demand now? It would be useful then to theorise the artist or producer to audience connection and, fortunately, Born (2005: 24-28) also described three ways this connection can be made (although she was discussing performer and audience):

1. Directly with the audience: but with recorded music there is no »direct « communication as such. As Evan Eisenberg describes it: "One of the paradoxes of the recording situation, namely that [for the performer] the audience is not there [is] the flip side of the fact that, for the listener, the performer is not there (Eisenberg 2005: 157).

2. To an idealized future or hypothetical audience: although in many ways, Eisenberg's paradox of recording means that any audience for recorded music is hypothesised rather than experienced.

3. To and from a synthesized / meta-audience: Born has in mind such phenomena as the focus group or an audience mediated through a record company which, again, seems a different form of hypothesised audience.

Eisenberg's paradox of recording and the way that it inflects Born's theories of distributed agency in relation to recorded music also impact on a systems theory approach. The audience is a substantial part of both the cultural domain and the social field: establishing valid rules of practice - for example through notions of authenticity, and judging creative output - not least through purchasing power, the power to bestow professional status on artists by enabling them to earn a living.

\section{Interpretation}

We have already looked at the idea of subject position and forcing the listener into a particular perspective to influence their interpretation. I want to extend this notion of a deliberately skewed or manipulated perspective towards the notion of creating a schematised representation of a given event or experience. Allan Moore (e.g. Moore 2009 and forthcoming 2011-12) and others have utilised the concept of conceptual blending to accommodate features such as multiple forms of ambience in the same recording i.e. the impossible situation of being in two different spaces at 
the same time. This process of blending involves the creation of a schematic representation of a situation that incorporates characteristics from several sources. Further to this, when we create a schematic representation that accentuates particular features over others we are also influencing the possible interpretation by an audience. If I make a line drawing of a cube I am accentuating the boundary lines above the texture of the surfaces, the way it is lit etc. In sound and recorded music there are also many examples of what we might call »cartoon « versions of environments that are used to suggest some features of, for example, particular venue types. In Florence \& The Machine's »You've Got The Love« (2009), the sound of a large venue is suggested through a »shorthand « of features that suggest certain acoustic characteristics whilst avoiding others. The »real« acoustic properties that would be experienced when hearing a band playing in a large hall would involve a long reverberation time on all of the instruments and a disproportionate exaggeration of the low frequency content because higher frequency reverberation dies away more quickly than lower frequencies. The fact that this accentuation of low frequencies comes from reverberation also means that the clarity of rhythmic and pitch information becomes muddied. The »cartoon « version of concert sound that is found in this example involves longer reverberation and a higher mix of ambient versus direct sound that is only added to higher frequency instrumental components such as the piano. At the same time the exaggeration of the bass frequencies is achieved without adding reverberation to lower frequency sounds by using a sustaining synthesiser bass sound rather than a bass guitar and by using compression. This provides the suggestion of a large space without the muddiness that the bass heavy reverberation of real life creates: a »cartoon « sound that affects our interpretation in much the same way as subject position manipulations.

\section{Conclusions}

Aside from presenting a theoretical model for analysis built from ecological perception, embodied cognition, metaphor and systems theory, this article also proposes that the theoretical gap between a creator's intentions and an audience's interpretations can be bridged through two basic mechanisms. The first is that, despite the intellectual heritage of postmodernism, there are aspects of our cognition of music that are universal to the extent that the shared experience of inhabiting, or rather being, a human body and utilising the human perceptual system in a world subject to consistent 
physical laws creates common experiences and common interpretations of some things. This was demonstrated through examples involving the perception of space and intimacy but can also be applied to fullness, warmth, levels and types of energy expenditure (and their emotional associations) and many other phenomena. The second mechanism involves the examination of culturally specific interpretations of some social phenomena through the idea of many layers of overlapping communities who share particular interpretations because of their specific environment, language, creative practice and social history. Within these communities, individuals can engage in centrifugal and centripetal forms of activity and interpretation and the notion of cultural domains and social fields allows us to both examine the influences at work within them and the ways in which change occurs. Thus, whilst an individual may work within a cultural domain of rules, conventions and traditions of creative practice, they may choose to conform to or reject those behavioural norms and, similarly, the social field that judges and provides critical comment on individual output can also be conformed to or rejected. This was demonstrated through examples in which particular types of technological mediation have acquired interpretative meaning for particular communities: the sound of scratching in DJ based dance music, the sound of lo-fi recording as a rejection of commercialism and so forth.

Of course, the major problem with this is of defining these communities, domains and fields. I may belong to one field as far as microphone techniques are concerned and to another in terms of mixing. I might be both a punk and an academic. I might share all the characteristics of a generic country music fan but have an irrational dislike of Dolly Parton's voice or the sound of pedal steel guitar. There's not only a problem with defining membership but also with the characteristics of the community. The domains and fields also imply a perfect or at least uniform system of information transmission for them to work i.e. that all of the members of a community have equal access to the rules and conventions of the domain and the judgments of the field. In short we're encountering all the problems of subjectivity that we were hoping to sidestep in the first place. However, the critical issue here is that these are conceptual models for thinking about the problem and not descriptions of »reality«. The question is not whether these communities, domains and fields actually exist so much as whether thinking about the world as if they exist gives us a better understanding of it. Indeed, in what is for me a very pleasingly recursive aspect of this, the analytical tools are very much like the thing they seek to analyse: they also provide a cartoon version of reality that highlights certain 
salient points in order to avoid the muddiness of reality and to suggest a particular interpretation of what is more important and what is less important.

\section{References}

Bahktin, Mikhail (1981). The Dialogic Imagination: Four Essays. Ed. by Michael Holquist. Austin, TX: University of Texas Press.

Born, Georgina (2005). »On Musical Mediation: Ontology, Technology \& Creativity.« In: 20th Century Music 2:1, pp. 7-36.

Clarke, Eric F. (2005). Ways Of Listening: An Ecological Approach to the Perception of Musical Meaning. Oxford, New York: Oxford University Press.

Csikszentmihalyi, Mihaly (1999). »Implications Of A Systems Perspective To The Study Of Creativity.« In: Handbook Of Creativity. Ed. by Robert J. Sternberg. New York: Cambridge University Press, pp. 313-335.

Eisenberg, Evan (2005). The Recording Angel. New York: McGraw Hill [1st ed. 1987].

Gibson, James (1979). The Ecological Approach to Visual Perception. Boston: Houghton Mifflin.

Lakoff, George \& Johnson, Mark (1980). Metaphors We Live By. Chicago: University of Chicago Press.

McIntyre, Phillip (2007). »Rethinking Creativity: Record Production and the Systems Model.« In: Art of Record Production Conference Proceedings, http://www. artofrecordproduction.com/content/view/210/95 (accessed February 28, 2011).

Monson, Ingrid (1996). Saying Something: Jazz Improvisation and Interaction. Chicago: University of Chicago Press.

Moore, Allan F. (2009) »Aspects of Embodied Meaning. « Unpublished paper presentation at the 2009 Art of Record Production conference at the Atrium, University of Glamorgan, Cardiff, November 15th, 2009.

Moore, Allan F. (forthcoming 2011/12) $\gg$ Beyond A Musicology Of Production «. In: The Art of Record Production: An Introductory Reader For A New Academic Field. Ed. by Simon Frith \& Simon Zagorski-Thomas. Farnham, Surrey: Ashgate Press.

Zagorski-Thomas, Simon (2008a). »The Musicology of Record Production. « In: 20th Century Music 4:2, pp. 189-207.

Zagorski-Thomas, Simon (2008b). »The Medium In The Message: Phonographic Staging Techniques that Utilize the Sonic Characteristics of Reproduction Media." In: Journal on the Art of Record Production 4, http://www. artofrecordproduction.com/component/option,com_docman/task,doc_view/gid ,81 (accessed February 28, 2011).

Zagorski-Thomas, Simon (2009). »What's Going On in What's Going On?« Unpublished plenary session paper at the International Association for the Study of Popular Music (IASPM) conference in Liverpool, July 13th, 2009.

Zagorski-Thomas, Simon (2010). »The Stadium In The Bedroom: Functional Staging, Authenticity and the Audience Led Aesthetic in Record Production. «In: Popular Music Journal 29:2, pp. 251-266. 


\title{
Discography
}

Darkthrone (1994). »Transilvanian Hunger.« On: Transilvanian Hunger. 12" vinyl album. Peaceville Records VILE43.

Drake, Nick (1970). »Thoughts Of Mary Jane.« On: Five Leaves Left. 12" vinyl album. Island Records ILPS 9105.

Florence \& The Machine (2009). »You've Got The Love.« On: Lungs. CD album. Universal Island Records 1797940.

Hannigan, Lisa (2009). »I Don't Know« On: Sea Sew. CD album. Hoop Recordings lhcd001.

Ke\$ha (2010). »Party At A Rich Dude's House.« On: Animal. CD album. RCA Records 88697640462.

Portishead (1994). »Biscuit.« On: Dummy. 12" vinyl album. Go! Beat Records 828 522-1.

The Rolling Stones (1969). "You Can't Always Get What You Want.« On: Let It Bleed. 12" vinyl album. Decca Records SKL 5025.

\begin{abstract}
This article proposes a methodological framework for the analysis of recorded popular music that draws upon ecological perception, embodied cognition, metaphor and systems theory. It further proposes that the theoretical gap between a creator's intentions and an audience's interpretations can be bridged through two basic mechanisms. The first is that the combination of the experience of embodiment in human form and the consistent physical laws of our environment provide common and consistent experiences and interpretations of certain phenomena. The second is that certain social phenomena can be examined through the notion of many layers of overlapping communities who share culturally specific interpretations based on their specific environment, language, creative practice and social history.
\end{abstract}

